S. Koizumi

Nagoya Math. J.

Vol. 60 (1976), 173-180

\title{
THE RING OF ALGEBRAIC CORRESPONDENCES ON A GENERIC CURVE OF GENUS $g$
}

\author{
SHOJI KOIZUMI
}

0. Let $p$ be a prime number or zero and let $g$ be a non-negative integer. Then there is a coarse moduli space $\boldsymbol{M}_{g}$ for complete nonsingular irreducible curves of genus $g$ defined over fields of characteristic $p$, which is an irreducible variety over the algebraic closure $\bar{F}_{p}$ of the prime field $F_{p}$. (Especially, $F_{0}$ is also denoted by $\boldsymbol{Q}$ as usual.) ([8], [2]). The curve corresponding to a generic point of $\boldsymbol{M}_{g}$ over $\bar{F}_{p}$ is called a generic curve of genus $g$. The purpose in this note is to prove the statement:

(I) the ring $\mathscr{E}(C)$ of algebraic correspondences on a generic curve $C$ is trivial, i.e., isomorphic to the ring $Z$ of rational integers.

Let $J\left(C^{\prime}\right)$ be a Jacobian variety associated to a complete non-singular irreducible curve $C^{\prime}$. As it is well-known, there is a canonical isomorphism between $\mathscr{E}\left(C^{\prime}\right)$ and the ring End $\left(J\left(C^{\prime}\right)\right.$ ) of endomorphisms of $J\left(C^{\prime}\right)$, and therefore the above (I) is equivalent to the following:

(II) we have, for a generic curve $C$,

$$
\text { End }(J(C)) \cong Z \text {. }
$$

Despite much plausibility of the result, as far as the author knows, no proof for our statement is found in any literature. ${ }^{(1)}$

The main tools for our proof are theories of moduli of curves and of reductions of abelian varieties at a discrete valuation. The proof for (II) is divided into two parts: first (1) the Jacobian variety $J(C)$ is simple, and secondly (2) using the simplicity of $J(C)$, the relation End $(J(C)) \cong Z$ is proved. When the genus is one, the first part (1) is trivial, and the second (2) is classical and well-known ([3]), while the statement (II) itself

Received March 3, 1975.

(1) Mr. Shigefumi Mori has kindly communicated to the author that he has examples of hyperelliptic curves of any genus and of any characteristic, each of which has the ring of algebraic correspondences isomorphic to $\boldsymbol{Z}$. 
is trivial in case $g=0$. The cases of higher genera are fairly easily deduced by induction on $g$, once one proves the simplicity of $J(C)$ in case $g=2$ and $=3$; and this is proved through dimensional arguments. In Section 1, we summarize what we quote from the moduli theory. We define the notion of the algebraic closure (or, briefly, a-closure) of moduli for a curve and for an abelian variety, which is less fine than the notion of the field of moduli for each ([6]), but fine enough for our use here. A difference of these two notions is described in the items 1.1.1 and 1.1.2, which will never be used throughout the paper. Section 2 is devoted to recalling some research from the reduction theory without explicit proofs. The proof of our result will be completed in Section 3. Acknowledgement must be given to the result of conversations with Mr. T. Sekiguchi which was useful for preparing this note.

\section{Preliminaries from moduli theory.}

We fix once for all, as "universal domain" a field $K$ of characteristic $p$, which is algebraically closed and of infinite transcendental degree over the prime field $F_{p}$. The fields which will be considered in the paper are all contained in $\boldsymbol{K}$. By a curve we always understand a complete non-singular irreducible curve defined over an algebraically closed field, except when the contrary is specifically stated.

PROPOSITION-DEFINITION 1.1. (2) Let $X$ be an abelian variety or a curve. Then there exists an algebraically closed field $K_{X}$ uniquely determined by $X$, which is characterized by the following two properties:

(ai) there is a variety $X^{\prime}$ isomorphic to $X$ which is defined over $K_{X}$

(aii) if a variety $X^{\prime \prime}$ is isomorphic to $X$ and defined over a field $K^{\prime}$, then the algebraic closure of $K^{\prime}$ contains $K_{X}$.

The field $K_{X}$ as above is called the "algebraic closure of moduli for $X$ " (or briefly " $a$-closure of moduli for $X$ ").

Proof. First, we remark that the uniqueness of $K_{X}$ is obvious from our conditions (ai) and (aii) once we prove the existence of it. We now use the terminology and results in [6]. In the case where $X$ is a curve, the algebraic closure of the field of moduli for $X$ satisfies the two

(2) Of course, we can discuss the notion of the "algebraic closure of muduli" for a more general structure, e.g., for an FM-structure in [6]. But we restrict ourselves only to the cases, with which we actually concern ourselves in this paper. 
conditions (ai) and (aii). In the case of abelian varieties, we take a very ample divisor $D$ on $X$. The pair $(X, \mathscr{A}(D))$ consisting of $X$ and the maximal algebraic family $\mathscr{A}(D)$, of positive divisors, containing $D$ defines an $\mathscr{A}$-Structure (or inhomogeneously polarized variety in the sense of [8]). The field of moduli for $(X, \mathscr{A}(D))$ exists ([6]), and as is easily seen, the algebraic closure $K_{X}$ of the field of moduli for $(X, \mathscr{A}(D))$ does not depend on $D$ but only on $X$, and has our properties (ai) and (aii). This completes the proof.

Remark 1.1.1. When we are concerned with the definition of the $a$-closure of moduli for a curve $X$, the curve $X$ does not have to be "complete and non-singular". In fact, if $X$ is an irreducible curve (perhaps not complete, with singularity), and if $X_{1}$ is a (complete nonsingular) curve birationally equivalent to $X$, then the $a$-closure $K_{X_{1}}$ of moduli for $X_{1}$ satisfies the two conditions $\left(a^{\prime} i\right)$ and $\left(a^{\prime} i i\right)$ on $X$, which are obtained, respectively, from (ai) and (aii) by substituting a "variety" and "isomorphic", respectively, by an "irreducible curve" (perhaps not complete, with singularity) and "birationally equivalent". On the other hand the hypothesis that a curve is complete and non-singular, is quite essential when we prove the existence of the field of moduli for the curve. The collection of irreducible curves (perhaps not complete, with singularity) satisfies the axioms of " $F M$-system" in the sense of ([6] p. 40) under the usual definitions of "fields of rationality" and of "transforms" of curves by field isomorphisms, and under "birational equivalence" in place of "isomorphy". The following Proposition 1.1.2 shows that the field of moduli for an irreducible curve $X$ in this $F M$-system does never exist if the universal domain is of characteristic $p>0$ and if the $a$ closure $K_{X}$ of moduli (which is defined as $K_{X_{1}}$ in the above), does not coincide with the algebraic closure $\bar{F}_{p}$ of $F_{p}$.

Proposition 1.1.2. Let $k^{\prime}$ and $k$ be two fields such that $k^{\prime}$ is a purely inseparably algebraic extension of $k$, and $K^{\prime}$ a regular extension of $k^{\prime}$. Then there is a field $K$ between $k$ and $K^{\prime}$ such that $K$ is regular over $k$ and $k^{\prime} K=K^{\prime}$.

Proof. ${ }^{(3)}$ It suffices to prove our assertion under the additional assumption that $\left[k^{\prime}: k\right]$ equals characteristic $p>0$ of $k$, i.e., $k^{\prime}=k(t)$ with $t \notin k$ and $t^{p} \in k$. Let $\left(x_{1}, x_{2}, \cdots, x_{n}\right)$ be a set of algebraically independent

(3) This proof is due to G. Shimura. 
elements of $K^{\prime}$ over $k^{\prime}$ such that $K^{\prime}$ is separably algebraic over $k^{\prime}\left(x_{1}, \cdots, x_{n}\right)$, and $K$ the set of all elements of $K^{\prime}$ separably algebraic over $k\left(x_{1}, \cdots, x_{n}\right)$. Then the field $K$ satisfies the conditions we required.

Q.E.D.

In the following we shall list some useful properties of the $a$-closure $K_{X}$ of moduli, most of which are directly derived from studies about curves and abelian varieties developed in these years. Let $\bar{F}_{p}$ be the algebraic closure of the prime field $F_{p}$. For the $a$-closure $K_{X}$ of moduli for $X$, we denote by $\operatorname{dim} K_{X}$ the transcendental degree of $K_{X}$ over $F_{p}$.

1.2. Let $M_{g}$ be a coarse moduli variety for curves of genus $g$ over fields of characteristic $p$. If $C$ is a curve of genus $g$, then the a-closure $K_{C}$ of moduli coincides with the algebraic closure $\overline{F_{p}(x)}$ of the field $F_{p}(x)$ generated over $F_{p}$ by the point $x$ of $\boldsymbol{M}_{g}$ corresponding to $C$. Furthermore, we have $\operatorname{dim} K_{C}=0$ (resp. $\leq 1$ or $\left.\leq 3 g-3\right)$ if $g=0$ (resp. $=1$ or $\geq 2$ ), and in each case $g>0$, the equality holds in the above inequality as to $\operatorname{dim} K_{C}$ if and only if $C$ is a generic curve.

Proof. From the definition of coarse moduli spaces ([8] p. 99), for any algebraically closed field $F$, there is a one-one correspondence between the set of all curves of genus $g$ over $F$ modulo isomorphism and the set of all $F$-rational points on $\boldsymbol{M}_{g}$. This proves $\overline{F_{p}(x)}=K_{C}$. As for the latter part, it is sufficient to show $\operatorname{dim} M_{g}=3 g-3$ (resp. $=1$ or $=0$ ) if $g \geq 2$ (resp. $=1$ or $=0$ ). In fact, this equality is classical in the case when $g=0$ or $=1$ ([4]). If $g \geq 2$, the coarse moduli space $\mathscr{M}_{g}$ for curves, of genus $g$, defined over fields of any characteristic is an irreducible scheme over $Z$ and we know that $\mathscr{M}_{g} \times \operatorname{Spec}(\overline{\boldsymbol{Q}})$ is an irreducible variety of dimension $3 g-3$, which is a coarse moduli variety of curves of genus $g$ over fields of characteristic zero. On the other hand, $\mathscr{M}_{g} \times \operatorname{Spec}\left(\bar{F}_{p}\right)$ coincides with $\boldsymbol{M}_{g}$, and has the same dimension as $\mathscr{M}_{g} \times \operatorname{Spec}(\bar{Q})$ because of the irreducibility of the scheme $\mathscr{M}_{g}$ ([8] p. 103, p. 143).

1.3. Let $C$ and $J(C)$ be a curve and its Jacobian variety, respectively. Then we have $K_{C}=K_{J(C)}$. In particular, if $X$ is a principally polarized abelian variety of dimension 2 , we have $\operatorname{dim} K_{X} \leq 3$.

The first equality $K_{C}=K_{J(C)}$ follows from Torelli's theorem, and the second inequality does from the above equality and the fact that every 
principally polarized abelian variety of dimension 2 is isomorphic to a Jacobian variety or the product of two elliptic curves ([15]).

Remark 1.3.1. If we use Mumford's theorem stated in [9], which says that all abelian varieties can be lifted to characteristic zero, we get an inequality $\operatorname{dim} K_{X} \leq \frac{1}{2} g(g+1)$ for any abelian variety $X$ of dimension $g$. Nevertheless, we have restricted our statement only to what we need for later use.

1.4. Suppose that the universal domain is of positive characteristic p. If $X$ and $Y$ are two isogenous abelian varieties of dimension $g$ and if $X$ is of p-rank $g$ (i.e., $X$ contains $p^{g}$ points of order $p$ ), we have $K_{X}=K_{Y}$.

Under the assumptions on $X$, there are only finitely many purely inseparable isogenies $X \rightarrow X^{\prime}$ of height one and in this case, we have $K_{X}=K_{X^{\prime}}$ ([10] p. 146-7). The equality about the a-closures of moduli for two isogenous abelian varieties $X$ and $X^{\prime}$ are more easily obtained in separable isogeny case without using the assumption on $p$-rank. Our general case is just a combination of the above two cases.

\section{Preliminaries from reduction theory.}

Now we recall some research from the reduction theory of abelian varieties. There are four items 2.1-2.4 we cite here: the first two 2.1 and 2.2 are fundamental and found in [14] or [7], the last one 2.4 follows from 2.3, and the third 2.3 will be reduced to a few well-known facts. Let $K$ be a field, and $v$ a discrete valuation of $K$.

2.1. Let $Y$ be an abelian subvariety of an abelian variety $X$, both defined over $K$. If the reduction $\tilde{X}$ of $X$ at $v$ is an abelian variety, then the reduction $\tilde{Y}$ of $Y$ at $v$ has an abelian subvariety $\bar{Y}$ of $\tilde{X}$ as its support.

2.2. Let $X$ be an abelian variety over $K$ such that all endomorphisms $\lambda$ in End $(X)$ are defined over $K$. If $X$ has an abelian variety $\tilde{X}$ as its reduction at $v$, we have a canonical injection: End $(X) \subseteq$ End $(\tilde{X})$ defined by $v$-reduction $: \lambda \mapsto \tilde{\lambda}$.

2.3. Let $J_{1}, J_{2}$ and $J$ be three Jacobian varieties associated to three independent generic curves, respectively, of genus $g_{1}, g_{2}$ and $g$, where 
$g=g_{1}+g_{2}$ and both $g_{1}$ and $g_{2}$ are positive. Then there exist three abelian varieties $J_{1}^{\prime}, J_{2}^{\prime}$ and $J^{\prime}$ such that $J_{1}^{\prime} \cong J_{1}, J_{2}^{\prime} \cong J_{2}$ and $J^{\prime} \cong J$, and that $J_{1}^{\prime} \times J_{2}^{\prime}$ is a reduction of $J^{\prime}$ at some discrete valuation.

2.4. Suppose that the universal domain is of characteristic $p>0$. If $C$ is a generic curve of genus $g$, then the Jacobian variety $J(C)$ of $C$ is of p-rank $g$.

The proof of the assertion 2.3 is directly reduced to the following three facts, each of which is known or easily proved.

2.3.1. If $C=C_{1} \cup C_{2}$ is a stable curve over an algebraically closed field, consisting of two curves $C_{1}$ and $C_{2}$ of genus $g_{1}$ and $g_{2}$ intersecting at only one point transversally (where both $g_{1}$ and $g_{2}>0$ from the definition of "stable curves"), then $C$ is of genus $g=g_{1}+g_{2}$ and a reduction of a curve $C^{\prime}$ of genus $g$ at some discrete valuation $v$ ([13]).

2.3.2. Let $C^{\prime}$ be a curve and let $C=C_{1} \cup C_{2}$ be a stable curve as in 2.3.1. If $C$ is the reduction of $C^{\prime}$ at a discrete valuation $v$, then there is a model $J\left(C^{\prime}\right)$ of the Jacobian variety of $C^{\prime}$ with $J(C)$ as its reduction at some extension $v^{\prime}$ of $v$, where $J(C)$ is a generalized Jacobian variety of $C$ ([2] Th. 2.5).

2.3.3. Let $C=C_{1} \cup C_{2}$ be a stable curve as in 2.3.1. Then the product $J\left(C_{1}\right) \times J\left(C_{2}\right)$ of Jacobian varieties of $C_{1}$ and $C_{2}$ is a generalized Jacobian variety of $C$ in a natural way.

As for 2.4, according to the previous 2.3 , the product $E_{1} \times \cdots \times E_{g}$ of $g$ generic elliptic curves $E_{i}$ is a reduction of an abelian variety $J^{\prime}$ isomorphic to $J(C)$. Since $E_{1} \times \cdots \times E_{g}$ is of $p$-rank $g$ and the $p$-rank does not increase under the process of reduction, we can conclude that $J^{\prime}$ and also $J(C)$ are of $p$-rank $g$.

\section{Main results.}

After all necessary preparations in the previous sections, our theorems will be proved straightforwardly.

THEOREM 3.1. The Jacobian variety $J(C)$ of a generic curve $C$ of genus $g \geq 1$ is simple.

Proof. The case when $g=1$ is trivial. In the cases of higher genera, if we assume $J(C)$ is not simple, $J(C)$ is isogenous to a product $X \times Y$ of two abelian varieties $X$ and $Y$ of lower dimensions. When 
$g=2$ or $=3$, we claim an inequality $(*) \operatorname{dim} K_{X \times Y}<\operatorname{dim} K_{J(C)}=3 g-3$. In fact if $g=2$, both $X$ and $Y$ are one dimensional and we have $\operatorname{dim} K_{X \times Y} \leq \operatorname{dim} K_{X}+\operatorname{dim} K_{Y} \leq 2<3$. If $g=3$ and $\operatorname{dim} X=2$, the inequality $(*)$ is a consequence of $\operatorname{dim} K_{X} \leq 3$. The latter inequality $\operatorname{dim} K_{X} \leq 3$ is obvious in the characteristic zero case. If the characteristic is positive, $J(C)$ is of $p$-rank 3 (cf. 2.4). Since $X \times Y$ isogenous to $J(C)$ is also of $p$-rank 3 , the $p$-rank of $X$ must be equal to 2 and $\operatorname{dim} K_{X} \leq 3$ because $X$ is isogenous to a principally polarized abelian variety (cf. 1.3, 2.4 and [10] p. 234). This proves the inequality (*) $\operatorname{dim} K_{X \times Y} \leq 3+1<6=\operatorname{dim} K_{J(C)}$. Since the inequality (*) contradicts 1.4 , we have finished the proof for the case $g=2$ or $=3$. Now we assume $g \geq 4$ and prove our theorem by induction on $g$. Assume that $J(C)$ is not simple and $X$ is a proper abelian subvariety of $J(C)$ of dimension $\leq g / 2$. Let $g_{1}$ and $g_{2}$ be two positive integers such that $g_{1}+$ $g_{2}=g$ and neither $g_{1}$ nor $g_{2}$ equals $\operatorname{dim} X$. In fact, such $g_{1}$ and $g_{2}$ can be taken, because $g \geq 4$. According to 2.3 , a reduction of $J(C)$ at some discrete valuation $v$ may be assumed to be the product $J_{1} \times J_{2}$ of the Jacobian varieties $J_{1}$ and $J_{2}$ of two generic curves, respectively, of genus $g_{1}$ and $g_{2}$. Then the support $\bar{X}$ of the reduction $\tilde{X}$ of $X$ at $v$ is an abelian subvariety of $J_{1} \times J_{2}$, at least one of whose projections to $J_{1}$ and $J_{2}$ must be a proper abelian subvariety of the component $J_{i}(i=1$, or $=2$ ). This contradicts the hypothesis of induction, which asserts the simplicity of $J_{i}$.

Q.E.D.

THEOREM 3.2. Let $J(C)$ be the Jacobian variety of a generic curve of genus $g \geq 1$. Then we have End $(J(C)) \cong Z$.

Proof. This will be proved by induction on $g$. If $g=1$, the result is well-known. In the general case, as in the proof of the previous theorem, we may assume that $J(C)$ has a product $J_{1} \times J_{2}$ of two Jacobian varieties $J_{1}, J_{2}$ of positive dimensions, as its reduction at some valuation $v$, such that End $\left(J_{i}\right) \cong Z$, for $i=1,2$ and that $J_{1}, J_{2}$ are not isogenous to each other (cf. 2.3). Then End $(J(C))$ is isomorphic to a subring of End $\left(J_{1} \times J_{2}\right)$ (cf. 2.2). On the other hand, End $(J(C))$ has no zero-divisor because $J(C)$ is simple, and End $\left(J_{1} \times J_{2}\right)$ is isomorphic to the ring $\boldsymbol{Z} \oplus \boldsymbol{Z}$. Hence, End $(J(C))$ must be isomorphic to $Z$ as a subring of $\boldsymbol{Z} \oplus \boldsymbol{Z}$, free from zero divisors.

Q.E.D. 
We end the paper with a few superfluous words: the proofs of our theorems need a good background and little originality.

\section{REFERENCES}

[1] Baily, W. L., Jr.: On the automorphism group of a generic curve of genus $g>2$, J. Math. Kyoto, 1 (1961), 101-108; Correction, 325.

[2] Deligne, P. and D. Mumford: The irreducibility of the space of curves of given genus, Publ. Math. I.H.E.S., No. 36 (Volume dedicated to O. Zariski), 1969, 75109.

[ 3 ] Deuring, M.: Die Typen der Multiplikatorenringe elliptischer Funktionenkörper, Abh. Math. Sem. Univ. Hamburg, 14 (1941), 197-272.

[4] Deuring, M.: Invarianten und Normalformen elliptischer Funktionenkörper, Math. Zeitschr., 47 (1941), 47-56.

[ 5 ] Grothendieck, A.: Séminaire de géométrie algébrique, 1960/61, Exporé III.

[6 ] Koizumi, S.: The fields of moduli for polarized abelian varieties and for curves, Nagoya Math. J., 48 (1972), 37-55.

[ 7 ] Koizumi, S. and G. Shimura: On specializations of abelian varieties, Scientific Papers of College of General Education, Univ. of Tokyo, 9 (1959), 187-211.

[ 8 ] Mumford, D.: Geometric invariant theory, Erg. d. Math., Neue F. Bd. 34, Springer Verlag, 1965.

[9] Mumford, D.: Bi-extensions of formal groups, Algebraic geometry, Tata Inst. Fund. Research, Bombay, Oxford Univ. Press, 1969, 307-322.

[10] Mumford, D.: Abelian varieties, Tata Inst. Studies in Math., 5, Oxford Univ. Press, 1970.

[11] Oort, F.: Finite group schemes, local moduli for abelian varieties, and lifting problems, Comp. Math., 23 (1971), 265-296 (also: Algebraic Geometry, Oslo, 1970, Wolters-Noordhoff, 1972.)

[12] Oort, F. and K. Ueno: Principally polarized abelian varieties of dimension two or three are Jacobian varieties, Jour. Fac. of Sci., Univ. of Tokyo, Sec. IA, 20 (1973), $377-381$.

[13] Schlessinger, M.: Functors of artin rings, Transact. Amer. Math. Soc., 130 (1968), 208-222.

[14] Shimura, G. and Y. Taniyama: Complex multiplication of abelian varieties and its applications to number theory, Publ. of Math. Soc. of Japan, 1961.

[15] Weil, A.: Zum Beweis des Torellischen Satzes, Akad. Wiss. Göttingen, math.phys. Klasse, 1957, No. 2, 33-53.

[16] Weil, A.: Foundations of algebraic geometry, Amer. Math. Society, Providence, R. I., 1946, 2nd edition, 1962.

Tokyo University of Education 